\title{
Modelling Drawbeads with Finite Elements and Verification
}

\author{
B.D. Carleer ${ }^{a}$, P.T. Vreede ${ }^{b}$, P. Drent ${ }^{b}$, M.F.M. Louwes ${ }^{a}$, J. Huétink ${ }^{a}$ \\ ${ }^{2}$ University of Twente. P.O. Box 217, 7500 AE Enschede, the Netherlands
}

${ }^{b}$ Hoogovens Groep R\&D, P.O. Box 10000, 1970 CA IJmuiden, the Netherlands

Drawbeads are commonly used in deep drawing processes to control the flow of the blank during the forming operation. In finite element simulations of deep drawing the drawbead geometries are seldom included because of the small radii; because of these small radii a very large number of elements is required in 3-D simulations. To cope with this problem, a 2-D analysis of the drawbead has been performed and the calculated restraining force will be applied in the near future in 3-D simulations with an equivalent drawbead element. Modelling drawbeads by only applying an additional restraining force is not satisfactory. During the flow of the material through a drawbead, the strain distribution changes and the material usually becomes thinner. These effects must be incorporated in the equivalent drawbead element.

For the modelling of the drawbead a 2-D plane strain finite element model was developed. Several simulations were carried out to investigate the behaviour of the drawbead. Various geometries were investigated, the friction coefficient was varied and also the frictionless case was taken into account.

To verify the model an experimental set-up was built. An extensive set of drawbead geometries was used. The results are compared with the finite element calculations and the similarity is very satisfactory.

\section{NUMERICAL MODEL}

\subsection{Finite element formulation}

The simulations were carried out using the implicit finite element code Dieka which is developed at the University of Twente in co-operation with Hoogovens Corporate Research. This code has the possibility to use a mixed EulerianLagrangian formulation, a combination of the Updated Lagrangian and Eulerian formulation [1].

The Updated Lagrange formulation is commonly used in solid mechanics. The grid points are moving with the material. It can easily describe history dependent properties like strain hardening. Besides, free surfaces can be followed in a natural way. A restriction of the updated Lagrange formulation is the limited deformation range because the element mesh is likely to be distorted, resulting in loss of accuracy and convergence.

Fluid dynamic problems are commonly solved by using the Eulerian formulation, the material flows through a fixed grid. An advantage of this method is that the grid will never be distorted because of great displacements. To incorporate history dependance, material path lines have to be determined along which the evolution equations must be integrated. The shape of a free surface cannot change during the simulation.

To avoid the above mentioned problems with both the Lagrangian and Eulerian formulation a mixed Eulerian Lagrangian formulation is applied. In this formulation the locations of the nodal points are not updated according to the displacements of the material but are decoupled. Now it is possible to keep a neat mesh and describe free surfaces as well.

\subsection{Material modelling}

The Ludwik-Nadai relation is used to describe the stress-strain relation:

$$
\sigma=C\left(\epsilon+\epsilon_{0}\right)^{n}
$$

In case of a multi-axial stress-state a criterion is needed to determine at which combination of stresses the material switches from elastic to plastic deformations. A well-known yield criterion is 
the Von Mises criterion. In terms of the three principal stresses it can be written as:

$$
\phi_{v M}=\left(\sigma_{2}-\sigma_{3}\right)^{2}+\left(\sigma_{3}-\sigma_{1}\right)^{2}+\left(\sigma_{1}-\sigma_{2}\right)^{2}-2 \sigma_{\text {yield }}^{2}=0
$$

In the simulations presented here, isotropic hardening was assumed using the Von Mises yield criterion. In case of a plane strain state, the effect of the R-value can be included in the model by introducing a correction factor which is the ratio of a Hill-like plane strain stress and the Von Mises plane strain stress. For a cold rolled low carbon steel this correction factor is larger than 1. The Bauschinger effect is not included in the model. In reality a lower yield stress may be found when the material is loaded in a cyclic way as occurs in drawbeads.

\subsection{Contact description}

To describe contact between two bodies interface elements were used. These elements were connected to the two impenetrable bodies. If the elements are open, the contact elements have no function. When penetration occurs a normal force in the interface elements is generated. When the overlap increases the normal force increases proportionally.

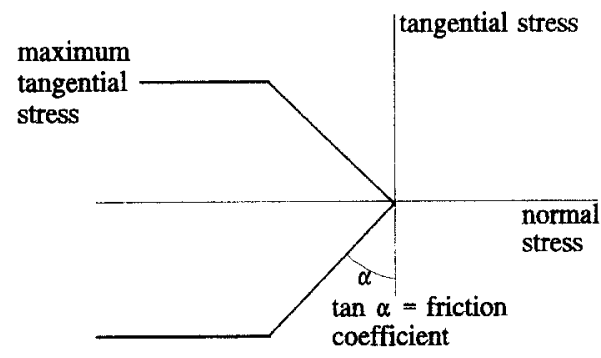

Fig. 1 The contact stress yield surface.

A tangential displacement will give a tangential stress. After a critical level of the tangential stress has been reached the bodies slide relative to each other. The stick-slip constitutive behaviour is introduced in a way which is analogous to the description of elasto-plasticity. In two dimensions, tangential and normal, this is:

$$
\phi_{C}=\tau-\mu \sigma_{n}
$$

where $\mu$ is the constant friction coefficient. The surface $\phi_{C}=0$ bounds the region in the contact stress space in which stick occurs. If sliding occurs the contact stress must satisfy the condition $\phi_{C}=0$.

\section{DRAWBEAD MODEL}

Two drawbead geometries were used, with dimensions according to figure 2 :
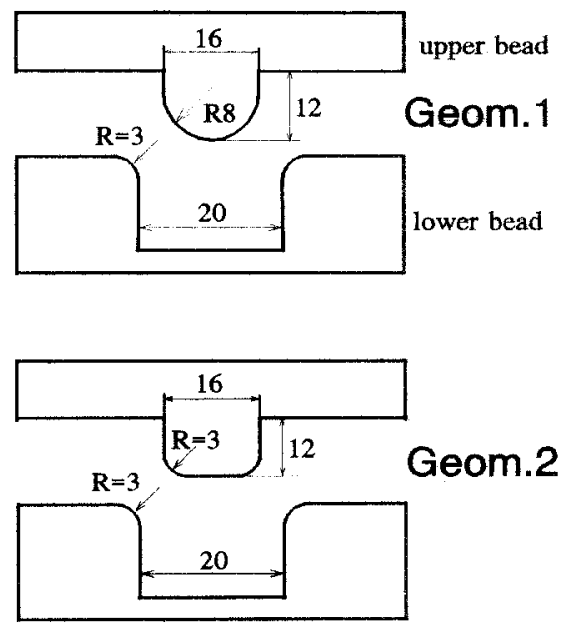

Fig. 2 Drawbead geometries.

Drawbeads can be loaded with an external (blankholder) force, however a constant clearance between the drawbeads which is larger than the sheet thickness is mostly applied in industrial practice, [3]. In all the following simulations a constant clearance of $0.9 \mathrm{~mm}$ is assumed.

In the simulations material properties of a low carbon steel were used:

Ludwik value $(C)=555 \mathrm{~N} / \mathrm{mm}^{2}$

$\mathrm{n}$-value $(\mathrm{n})=0.245$

initial yield stress $=160 \mathrm{~N} / \mathrm{mm}^{2}$

The blank thickness was $0.7 \mathrm{~mm}$.

\section{NUMERICAL RESULTS}

The sheet was modelled with four layers of four node plane strain elements. The total number 
of elements used was 800 .

Initially, the simulations were started with a flat blank (like in reality). Later on, it turned out that the calculation time could be reduced considerably by starting the calculation with a predeformed sheet in the drawbead, see figure 3 . Not modelling the initial closing of the drawbead had hardly influence on the results, [2], figure 4 gives an indication of this influence for the frictionless case.

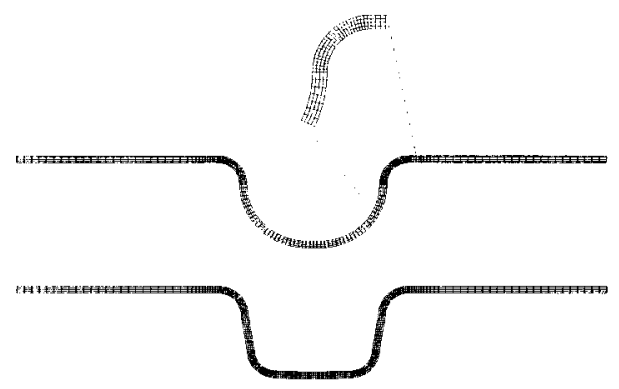

Fig. 3 Pre-deformed finite element meshes. The upper mesh (with magnified zone) is used in geometry 1 , the lower mesh is used in geometry 2.

In the simulations we used the mixed EulerianLagrangian formulation. The mesh is fixed in flow direction, we are an observer of the drawbead and we see how the sheet is pulled through the drawbead. In direction perpendicular to the flow the mesh is free to move. The advantage of this

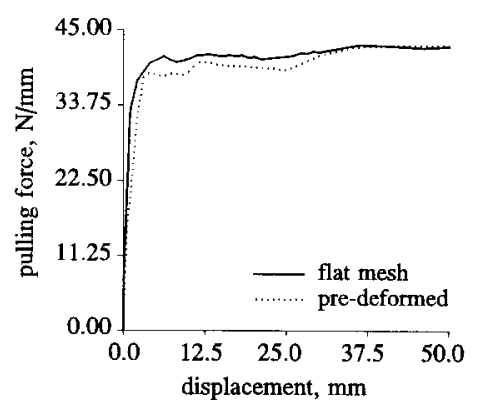

Fig. 4 Comparison of pulling force. The dotted line is the force found with the pre-deformed mesh, the solid line is found when a flat mesh is used. In both cases geometry 1 is considered. formulation is that the grid refinements remain at their place and the effects of sheet thinning can be described as well.

Figure 5 gives the calculated force to pull the strip through the drawbeads as a function of the strip displacement in case that friction is neglected.

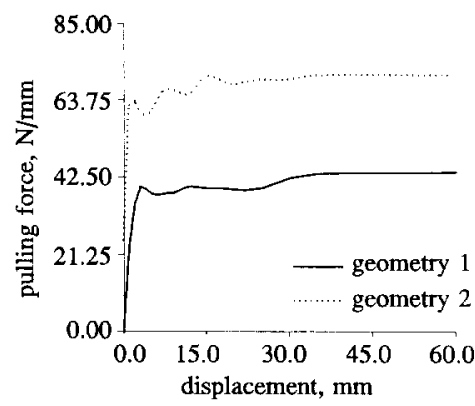

Fig. 5 Comparison of calculated pulling force. The upper (dotted) line is the force found with geometry 2, the lower (solid) line is found in case geometry 1 is considered.

The stationary solution was reached after pulling out the sheet $30 \mathrm{~mm}$. From now on we define the restraining force as the stationary pulling force.

The resistance of a strip to move through a drawbead is caused by friction and by bending and unbending of the strip. Let us first consider the frictional part. When we apply a constant clearance between the die and the blankholder the sheet and the tools only have contact in a few small zones. Contact between lower bead and sheet occurs at the small radii. In case of geometry 1 the contact zone of sheet and upper bead is relatively large, in case of geometry 2 the contact is more localised. The friction stresses for geometry 1 and 2 are given in figure 6 and 7 respectively; be aware of the different scales. A friction coefficient of 0.16 was applied in both cases. The sheet is pulled out from the left to the right.

The peaks in the contact shear stress are caused by the bending on the sheet. The right peak (exit side) is larger than the left peak because of extra tension in the strip.

In comparison with experiments simulations have the advantage that parameters can easily be changed. By changing the friction coefficient the 


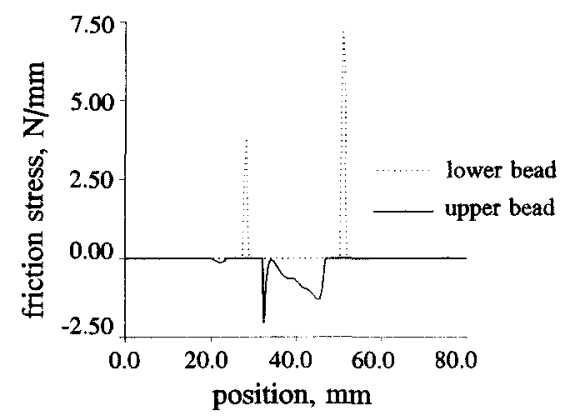

Fig. 6 Friction stress as a function of the position. Geometry 1.

influence of the friction can be studied. It is even possible to neglect the effect of friction by setting the friction coefficient zero. When there is no friction the restraining force is only caused by bending of the sheet. The restraining force as a function of the friction coefficient is shown in figure 8 . With increasing friction coefficient the restraining force increases more than linearly.

As a matter of fact, the effect of friction influences the required bending energy and vice versa, so these effects cannot be uncoupled.

When the sheet passes the drawbead it is being bent and stretched. Due to these deformations we find a tangential strain in the sheet. The tangential co-ordinate lies in the profile direction of the deformed sheet. Figure 9 and 10 show the tangential strain in the blank passing geometry 1 and geometry 2 respectively. In both cases a friction

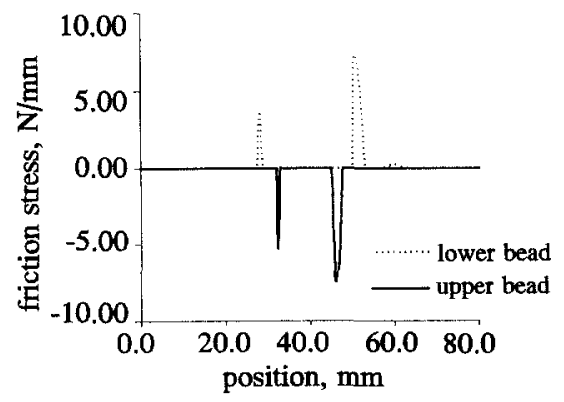

Fig. 7 Friction stress as a function of the position. Geometry 2.

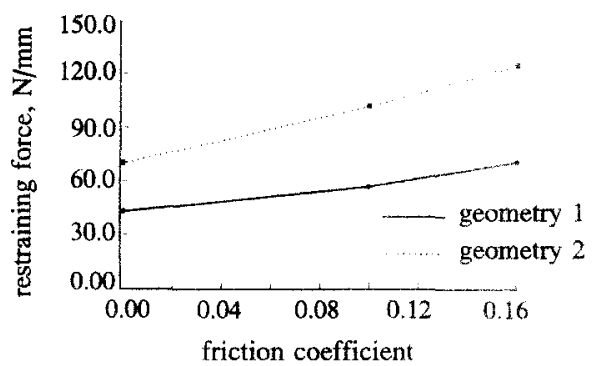

Fig. 8 Friction force as a function of the friction.

coefficient of 0.16 was applied. Tangential strains larger than zero indicate stretching, values smaller than zero indicate compression.

Focusing on geometry 1 , we observe three zones. The first zone represents bending around the first radius of the lower bead, then bending around the upper bead and at last bending around the second radius of the lower bead. Considering geometry 2 , we observe four distinctive zones. The inlet and exit zones show similar effects as in geometry 1. The zone of contact with the upper bead is split up now in two separate zones. Due to this additional bending the total tangential strain near the exit area when using geometry 2 is larger than the one of geometry 1 .

Since the plane strain state is assumed and the material is modelled to be almost incompressible, the thickness strain has the same value (except for the sign) as the tangential strain.

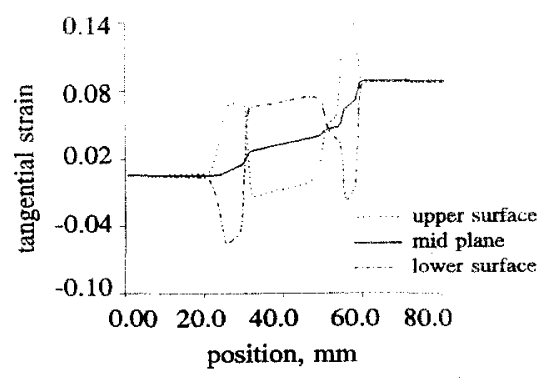

Fig. 9 Tangential strain distribution in the sheet at the sheet surfaces and the mid plane. Geometry 1. 


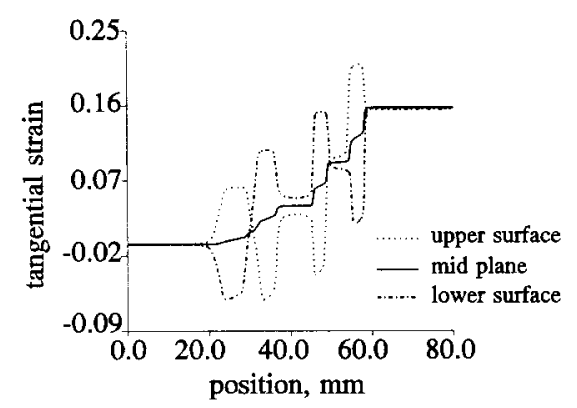

Fig. 10 Tangential strain distribution in the sheet surfaces and the mid plane. Geometry 2.

\section{EXPERIMENTAL VERIFICATION}

\subsection{Experimental set-up}

At Hoogovens Corporate Research the experiments were performed at a fully equiped Erichsen press. The tool was designed to exchange the beads allowing easy testing of several drawbead configurations. The experimental setup as well as the most relevant dimensions are depicted in figure 11. Significant geometrical difference between finite element calculation and experiment are the extra radii which is a consequence of using a deep drawing press. The surface roughness of the tools was about $0.25 \mu \mathrm{m}$.

All combinations of geometries and materials were tested with a range of (blank holder) forces. Besides, we studied the case of a constant clearance of $0.9 \mathrm{~mm}$ between the drawbeads, leaving $0.2 \mathrm{~mm}$ space between sheet and beads.

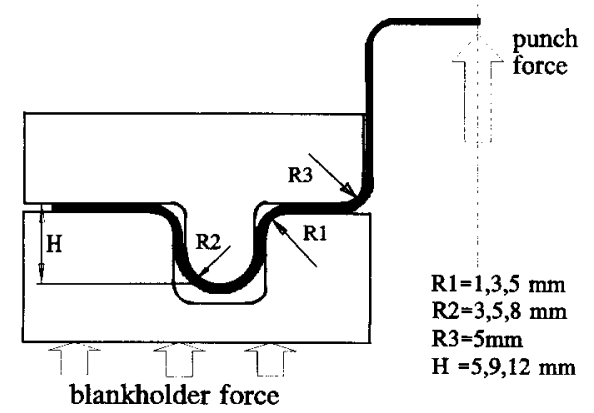

Fig. 11 Experimental setup of the drawbead tester.
The material used in this study was a deep drawing steel with mechanical properties as listed in section 2, "drawbead model". The dimensions of the blanks were $50 * 500 \mathrm{~mm}$. Throughout the drawing experiment the blanks were prepared with the long side oriented in rolling direction.

The applied lubricant was a deep drawing oil with a viscosity of 0.04 Pas at room temperature. The blanks were lubricated on both sides with help of a paint roller. The punch speed was kept constant at $3 \mathrm{~mm} / \mathrm{s}$.

\subsection{Experimental results}

In figure 12 the measured pulling force in case of geometry 1 and 2 is printed. We cannot compare this figure directly with figure 5 since two extra radii are in the experimental setup compared with the finite element analysis. Besides, the results in figure 5 are found assuming no friction.

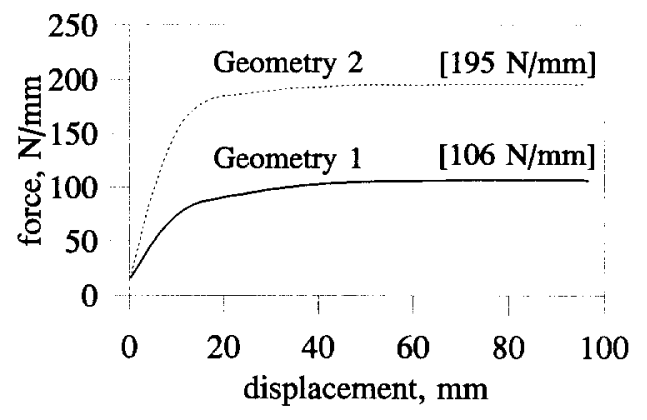

Fig. 12 Measured pulling force, The upper dotted line concerns geometry 2, the lower solid line was found using geometry 1.

After performing the experiments the thicknesses were measured. The measuring locations of the strip can be found in figure 13. The results of the thickness measurements are plotted in figure 14. As already mentioned, the thickness strain has the value with opposite sign of the tangential strain, assuming plane strain and neglecting elastic effects. Measuring point 6 gives the strain which can be compared with the finite element results. From figure 14 we can conclude that measuring points 8,9 and 10 have not passed the drawbeads entirely. 


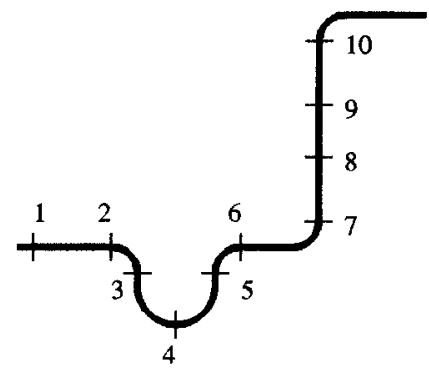

Fig. 13 Locations of measuring points after drawing.

\subsection{Results evaluation}

To compare the measured results with the finite element calculations, an additional $90^{\circ}$ bend was calculated for the stationary restraining force. The restraining force found in the drawbead calculations was applied as a load on the $90^{\circ}$ bend. The initial conditions of the $90^{\circ}$ bend (changed thickness and equivalent strain distribution) were obtained from the drawbead calculations described in section 3. The total force, which can be compared with the measured punch force, is collected in table 1 .

The calculated tangential strain after passing geometry 1 and 2 was 0.085 and 0.16 , see figure 9 and 10 . We measured a thickness strain of -0.08 and -0.19 at location 6 , figure 14 . More accurate predictions may be found when better constitutive descriptions and more reliable friction laws are applied.

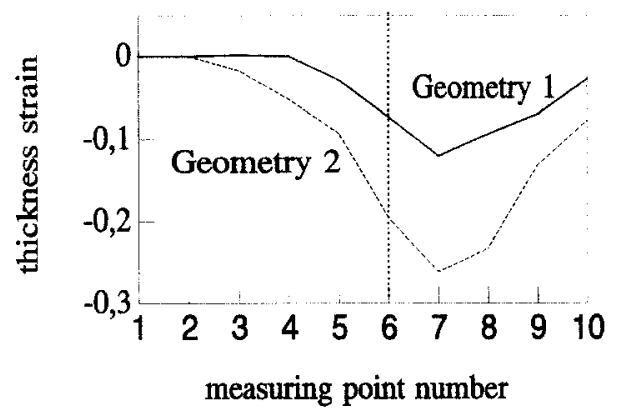

Fig. 14 Measured thickness strain of strips after passing drawbead geometry 1 (solid line) and 2 (dotted line). The vertical line indicates the exit of the drawbead.
Table 1 Comparison of the pulling force [N/mm]

\begin{tabular}{|l|l|l|l|l|}
\hline geom. & $\mu$ & $\begin{array}{l}\text { FE result } \\
\text { drawbead }\end{array}$ & $\begin{array}{l}\text { FE result } \\
\text { drawbead } \\
+90^{\circ}\end{array}$ & $\begin{array}{l}\text { Mea- } \\
\text { sure- } \\
\text { ment }\end{array}$ \\
\hline 1 & 0 & 43 & 55 & \\
& 0.1 & 57 & 80 & 106 \\
& .16 & 71 & 105 & \\
\hline \multirow{2}{*}{2} & 0 & 70 & 92 & \\
& 0.1 & 102 & 142 & 195 \\
\hline
\end{tabular}

\section{CONCLUSIONS}

In this article a finite element model to study the effects of drawbeads has been given and the results were compared with experiments. The similarity of the results was reasonable, however the friction coefficient was unknown. The assumed coefficient of 0.16 is a realistic value in sheet metal applications. With this assumption the measured thickness strain agrees fairly well with the calculated results. We conclude that the present finite element model is a reliable tool to predict the effect of drawbeads. In the near future these results will be incorporated in 'drawbeadelements' in a 3-D deep drawing model. Not only the restraining force need to be included, also the thinning and additional (equivalent) deformation must be taken into account. [4], [5]

\section{REFERENCES}

1. Huétink, J, On the simulation of thermo-mechanical forming processes, Dissertation, University of Twente, 1986, The Netherlands.

2. Beugels, J, Masters thesis, University of Twente, 1993, The Netherlands. (report in Dutch)

3. Einflieswulste und Ziehstäbe in Stanzerei-Gro $\beta$ werkzeugen, VDI-Richtlinien nr 3141, April 90

4. Cao, J ea, Drawbead penetration as a control element of material flow, SAE 930517 , SP-944

5. Taylor, L. ea, Numerical Simulations of sheet Metal Forming, Numisheet '93 conference proceedings, edited by E. Nakamachi ea 